\title{
Holistic Health improvement using the Internet of Bio-Nano Things Based Treatment
}

\author{
Shifa H. Sarker \\ Longfield - Davidson Heights \\ Nepean, ON K2J 5C6, Canada \\ shoss12@ocdsb.ca
}

\begin{abstract}
The Internet of Bio-Nano Things (IoBNT) is rapidly making its way into becoming a leading method of technology used in the treatment of health issues for the next generation. Today's healthcare sector places a tremendous amount of emphasis on the diagnoses and treatments of the symptoms and conditions of physical illnesses. However, the wellbeing of one's psychological state is largely ignored - despite past researches indicating it to play a tremendous role in the formation and continuity of a vast variety of physical illnesses. This indicates that, in order to ensure that measures for the wellbeing of an individual have been optimized, the healthcare provided to them must take on a holistic approach, focusing on treating both their physical and psychological illnesses. In methods of treatments that are heavily relied on today, an individual's physical and psychological wellbeing are observed and considered as independent variable. However, researches conducted in recent years have indicated that, due to a wide range of factors including the massive presence of genetically modified structures in our diets and increased use of advanced technological items in our daily lives, the physical and psychological illnesses of an individual are increasingly correlated. In this paper, the holistic treatment of health using future IoBNT technology has been studied, and how the correlated factors of physical and psychological wellbeing can be manipulated using IoBNT to maximize measures of providing the best treatments to patients. The results presented in this paper show that the probability of holistic healthiness of a person is very low when conventional methods of treatment typically used today are consulted and how a person's probability of holistic healthiness can be kept at a very high level using the nanotechnology-based treatment such as IoBNT.
\end{abstract}

Keywords: Bio-Nano Things, Health, Holistic, Illness, Internet, Physical, Psychological.

\section{Introduction}

Currently, the holistic healthiness of a person is defined as the state of being free of physical and psychological illnesses. It is expected that the Internet of Bio-Nano Things (IoBNT) based health treatment will be used widely to diagnose not only a wide variety of physical diseases as it already does today [1], [2], [3], but nanotechnology will rapidly become a tool for psychological treatments as well [4].

Many mathematical models have been presented only for physical treatments, few of them being [2], [3], and [5]. The mathematical models for psychology have also been presented in several other papers, one of them being [6]. For the vast majority of the time, the mathematical models for physical treatment and psychological treatment are shown separately. In this paper, we have considered not only holistic health treatment using physical treatment and psychological treatment also the effects that follow these correlated factors. 


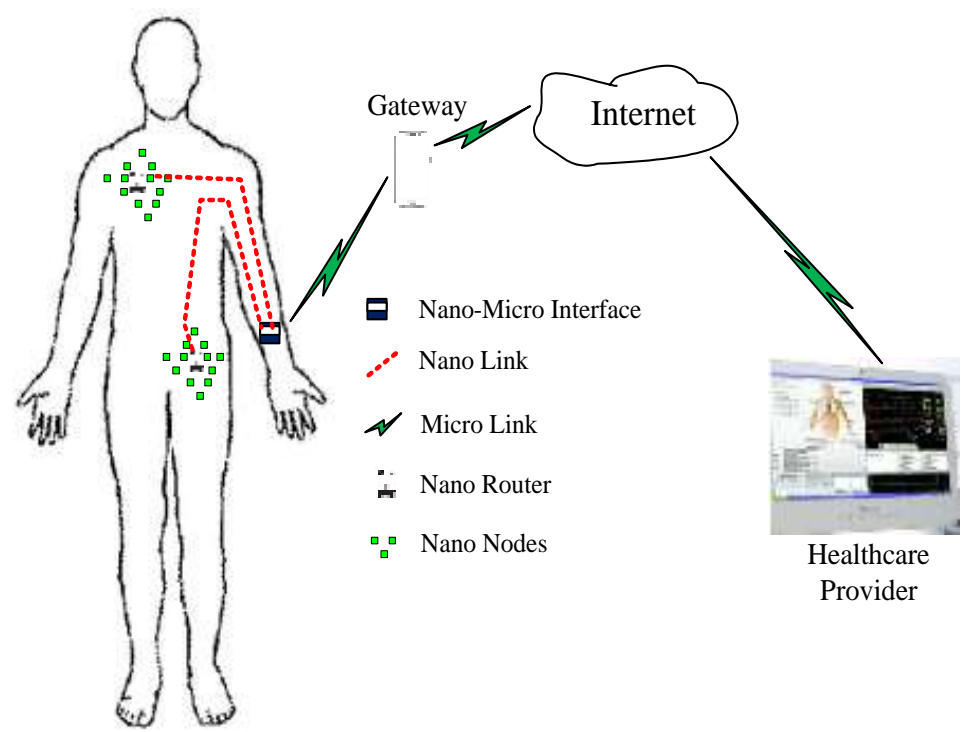

Fig. 1: Simplified network architecture for the Internet of Bio-Nano Things (IoBNT) based treatment.

In this paper, we have considered the Internet of Bio-Nano Things (IoBNT) based holistic health treatment as shown if Figure 1. Nanotechnology based molecular communication take place inside a human body, where it establishes nano links among nano nodes, and one or more nano-routers may help in the molecular communication. A nano-micro interface is used for converting from nano signal to micro signal and vice versa. The nano-micro interface sends typically the micro wireless signal to the gateway, which subsequently will send the signal to the Internet. This permits the healthcare provider to receive the information anywhere in the world where the Internet is available, even if they are in significantly distant locations from their patient.

\section{Modelling a Person's Either Only Physical or Only Psychological health condition}

Let us consider a specific person's health condition, which is consists of two parts: physical and psychological. Consider the overall health condition as a ship. If a patient is physically ill but psychologically healthy, then the overall health condition of the individual can be analogous to Fig. 2 (a). On the other hand, if the patient is psychologically ill but physically healthy, then the overall health condition can be described similar to Fig. 2 (b). For a healthy person, he/she should be physically and psychologically healthy as shown in Fig 2. (c).
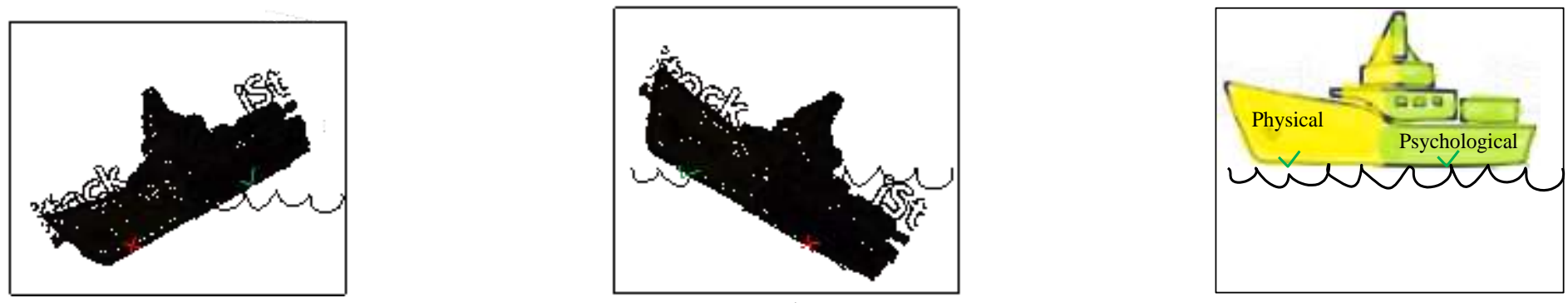

Fig. 2: One person's analogy of health condition.

\subsection{Mathematical Model Considering only Physical IIIness}

Consider that a person becomes physically ill with a rate of $\lambda 1$. Assume that a person became three consecutive times physically ill after 3, 4 and 5 months respectively. Thus, the average rate of becoming physically ill $\lambda 1$ is equals to per 4 months, or per 120 days. On average that person is becoming physically ill after every 120 days. Assume that the recovering rate is also exponential and independent. The average illness time is $1 / \mu 1$. For example one person ware three times ill for 6 days, 7 days and 8 days respectively. Therefore, the average physical illness duration $1 / \mu 1$ is equals to 7 days. If the physical illness intensity is $\rho 1$, then we can write 


$$
\rho 1=\frac{\lambda 1}{\mu 1}
$$

A list of parameters used in this paper is given in Table 1. Consider an example of physical illness intensity. The average rate of becoming physically ill $\lambda 1$ is equals to per 4 months, or per 120 days. If the average physical illness duration $1 / \mu 1$ is equals to 7 days, then the average illness intensity $\rho 1$ is equals to $[\{1 /(120$ days $)\} * 7$ days $]=$ 0.05833, which is dimensionless. According to the Fig. 3 (a), the probability that a person will become physically ill with the probability of

$$
Q_{P}=Q_{P H} \lambda 1 \frac{1}{\mu 1}=Q_{P H} \rho 1
$$

Considering only physical illness, the addition of only two probabilities will be equals to one. Mathematically,

$$
Q_{P}+Q_{P H}=1
$$

The probability that a person will be healthy considering only physical illness, can be written from Eqs. (2) and (3), as

$$
Q_{P H}=(1+\rho 1)^{-1}
$$

Fig. 3 (b), shows the numerical results of healthiness probability, $Q_{P H}$, with the variation of the physical illness intensity, $\rho 1$, when only physical illness is considered. Clearly, the physical healthiness probability, $Q_{P H}$, decreases with the increase of physical illness intensity, $\rho 1$.

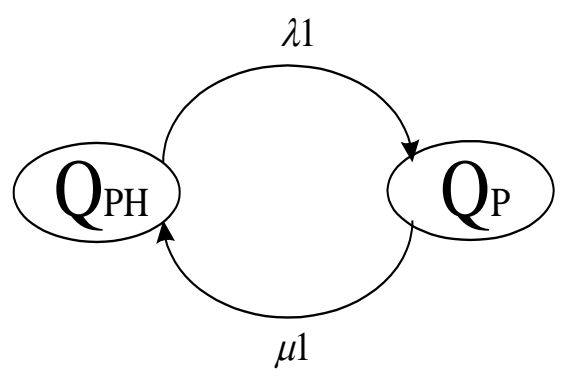

(a)

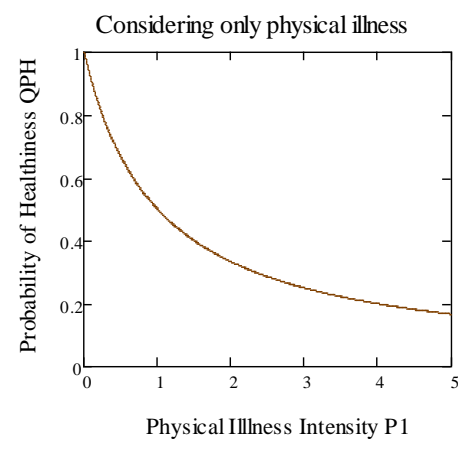

(b)

Fig. 3: (a) Markov model considering only physical illness; (b) The probability of healthiness, considering only physical illness.

Table 1: List of parameters used in this paper.

\begin{tabular}{|c|l|c|l|}
\hline Parameter & \multicolumn{1}{|c|}{ Description } & Parameter & \multicolumn{1}{|c|}{ Description } \\
\hline$\lambda 1$ & Average rate of becoming physically ill & $t 1$ & Physically illness treatment correlation factor \\
\hline $1 / \mu 1$ & Average physical illness time & $\beta$ & Psychologically illness correlation factor \\
\hline$\rho 1=\lambda 1 / \mu 1$ & Physically illness intensity & $t 2$ & Psychologically illness treatment correlation factor \\
\hline$\lambda 2$ & Average rate of becoming psychologically ill & $f$ & Physically and psychologically same illness correlation factors \\
\hline $1 / \mu 2$ & Average psychological illness time & $t$ & Physically and psychologically same treatment correlation factors \\
\hline$\rho 2=\lambda 2 / \mu 2$ & Psychologically illness intensity & $n$ & IoBNT based treatment correlation factor \\
\hline$\alpha$ & Physically illness correlation factor & $Q_{H n}$ & Probability that a person is healthy with IoBNT based treatment \\
\hline
\end{tabular}




\subsection{The Probability of Healthiness Considering only Psychological IIIness}

Let us consider that a person becomes psychologically ill with a rate of $\lambda 2$. Assume that a person became three consecutive times psychologically ill after 1 year two months, 1 year four months and 1 year six months respectively. Thus, the average rate of becoming psychologically ill $\lambda 2$ is equals to per 1 year four months, or per 485 days. In other words, on average that person is becoming psychologically ill after every 485 days. Assume that the recovering rate is also independently and exponentially distributed. Assume that the average psychologically illness time is $1 / \mu 2$. For example one person ware three times psychologically ill for 7 months, 8 months and 9 months respectively. Therefore, the average psychological illness duration $1 / \mu 2$ is equals to 8 months or 320 days. If the psychological illness intensity is $\rho 2$, then we can write

$$
\rho 2=\frac{\lambda 2}{\mu 2}
$$

Consider an example of psychological illness intensity. The average rate of becoming psychologically ill $\lambda 2$ is equals to per 1 year 4 months, or per 485 days. If the average psychological illness duration $1 / \mu 2$ is equals to 320 days, then the average psychological illness intensity $\rho 2$ is equals to $\left[\{1 /(485 \text { days })\}^{*} 320\right.$ days $]=0.6598$. Please note that the psychological illness intensity $\rho 2$ is unit-less. According to the Fig. 4 (a), the probability that a person will become psychologically ill with the probability of

$$
Q_{S}=Q_{S H} \lambda 2 \frac{1}{\mu 2}=Q_{S H} \rho 2
$$

Considering only psychological illness, the addition of only two probabilities will be equals to one. We can write,

$$
Q_{S}+Q_{S H}=1
$$

The probability that a person will be healthy considering only physical illness, can be written from Eqs. (6) and (7), as

$$
Q_{S H}=(1+\rho 2)^{-1}
$$

Fig. 4 (b), shows the numerical results of healthiness probability, with the variation of the psychological illness intensity, $\rho 2$, when only psychological illness is considered. Certainly, the psychological healthiness probability, $Q_{S H}$, decreases with the increase of psychological illness intensity, $\rho 2$.

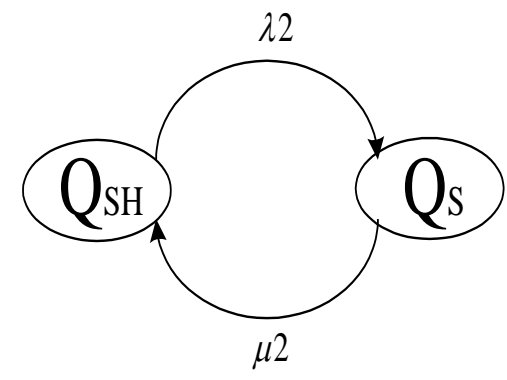

(a)

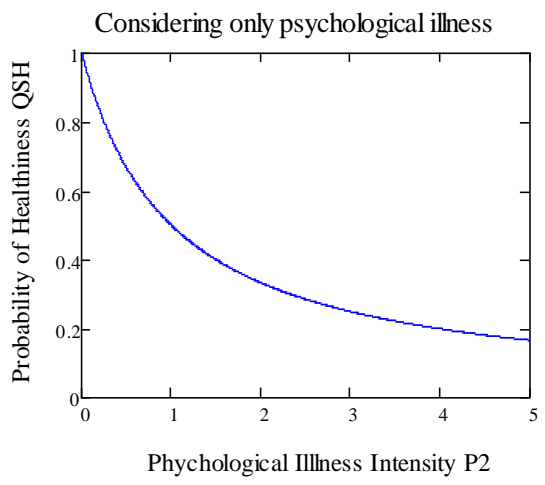

(b)

Fig. 4: (a) Markov model considering only psychological illness; (b) The probability of healthiness, considering only psychological illness. 


\section{Holistic Healthiness Considering Physical IIIness and Psychological IIIness}

Now let us consider the holistic healthiness taking into account physical illness and psychological illness together. Let a person becomes physically ill is exponentially distributed with a rate of $\lambda 1$. After becoming physically ill, he or she become also psychologically ill with the same rate of $\lambda 1$. Due to the excessive use of technology in our food and in our daily life, a person becomes $\alpha$ times more than that of the rate of $\lambda 1$, where $\alpha \geq 1$ (Figure 5). Excessive use of technology not only has disadvantages, it has also advantages to keep a person healthy. Because of use of advanced new technologies, a person's illness rate will be decreased by $t 1$ times, where $t 1 \geq 1$. Considering these two correlation factors $\alpha$ and $t 1$, the overall illness rates are $\frac{\alpha}{t 1} \lambda 1$. Assume that the recovering rate is also exponential and independent with a rate of $\mu 1$. The probability that a person will become first physically ill is

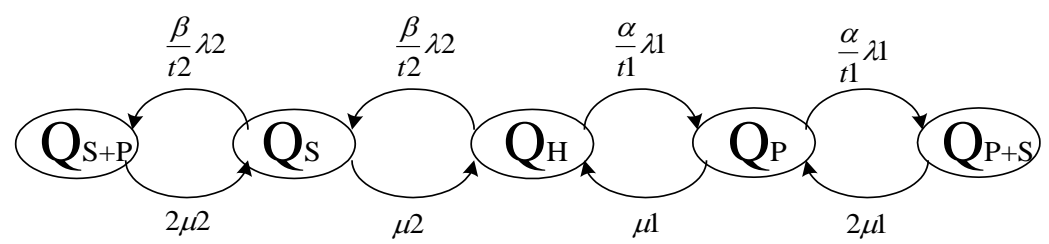

Fig. 5: The probability of healthiness, considering co-related physical and psychological illnesses and treatment.

$$
Q_{P}=Q_{H} \frac{\alpha \lambda 1}{t 1} \frac{1}{\mu 1}=Q_{H} \frac{\alpha \rho 1}{t 1}
$$

The probability that a person will become first physically ill the psychologically ill can be written as

$$
Q_{P+S}=Q_{P} \frac{\alpha \lambda 1}{t 1} \frac{1}{2 \mu 1}=Q_{P} \frac{\alpha \rho 1}{2 t 1}=Q_{H} \frac{1}{2}\left(\frac{\alpha \rho 1}{t 1}\right)^{2}
$$

Similarly, a person can become psychologically ill is

$$
Q_{S}=Q_{H} \frac{\beta \lambda 2}{t 2} \frac{1}{\mu 2}=Q_{H} \frac{\alpha \rho 2}{t 2}
$$

Using the same procedure, the probability that a person will become first psychologically ill and then physically ill the can be written as

$$
Q_{S+P}=Q_{S} \frac{\beta \lambda 2}{t 2} \frac{1}{2 \mu 2}=Q_{S} \frac{\beta \rho 2}{2 t 2}=Q_{H} \frac{1}{2}\left(\frac{\beta \rho 2}{t 2}\right)^{2}
$$

In steady state, the addition of all five probabilities shown in Fig. 5 is equals to one. Mathematically, we can write

$$
Q_{H}+Q_{P}+Q_{P+S}+Q_{S}+Q_{S+P}=1
$$

Using the values given in Eqs. (9) to (12) and setting those values into Eq. (13), we can evaluate the probability that a person will be holistic healthy with the probability of

$$
Q_{H}=\left[1+\left(\frac{\alpha \rho 1}{t 1}\right)+\frac{1}{2}\left(\frac{\alpha \rho 1}{t 1}\right)^{2}+\left(\frac{\beta \rho 2}{t 2}\right)+\frac{1}{2}\left(\frac{\beta \rho 2}{t 2}\right)^{2}\right]^{-1}
$$


Let us consider a special case where the illnesses are happening in uncorrelated manner. Then we can write $\alpha=\beta=1$. If the patient is using normal treatment, not advanced technology treatment, then $t 1=t 2=1$. The illness intensities are also equal; i.e., $\rho 1=\rho 2=\rho$. Setting these values into Eq. (14), the probability that a person will be healthy with physical and psychological illnesses is

$$
Q_{H B}=[1+\rho]^{-2}
$$

The numerical results of Eqs. (4), (8) and (15) is depicted in Fig. 6. Fig. 6 shows the holistic healthiness comparison between physical \& psychological illnesses, $Q_{H B}$, only physical illness, $Q_{P H}$ and only psychological illness, $Q_{S H}$. Clearly, the probability of holistic healthiness decreases, comparing with either only physical illness or only psychological illness.

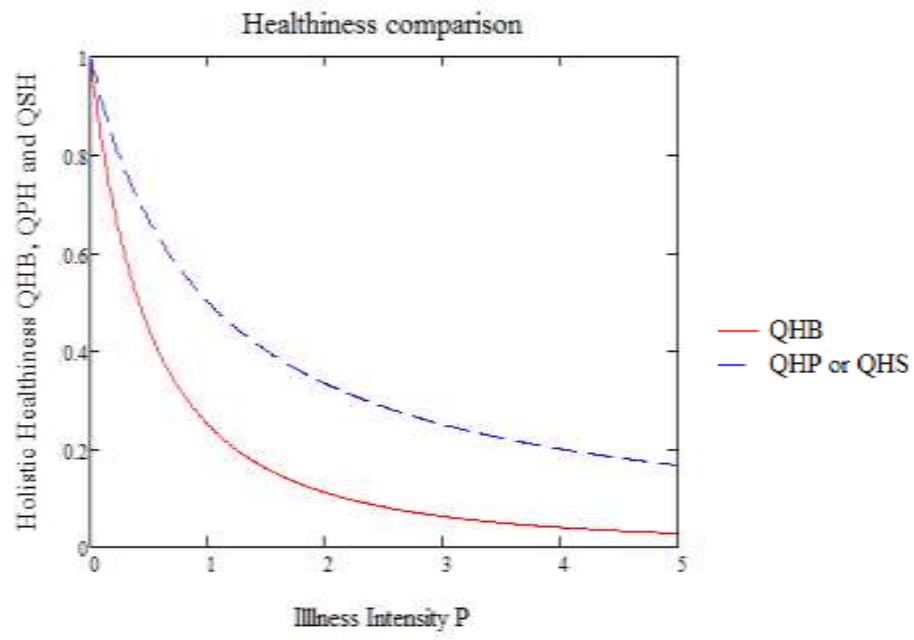

Fig. 6: The healthiness comparison between physical \& psychological illnesses, $Q_{H B}$, only physical illness, $Q_{P H}$ and only psychological illness, $Q_{S H}$.

Consider now another special condition, where the illnesses are happening in correlated manner, with equal co-relation factors. Then we can write $\alpha=\beta=f$. The illness intensities are also equal; i.e., $\rho 1=\rho 2=\rho$. Setting these values into Eq. (14), the probability that a person will be healthy with equal correlated factors of physical and psychological illnesses; but without any treatment is

$$
Q_{H}=\left[1+\left(\frac{f \rho}{t}\right)+\frac{1}{2}\left(\frac{f \rho}{t}\right)^{2}+\left(\frac{f \rho}{t}\right)+\frac{1}{2}\left(\frac{f \rho}{t}\right)^{2}\right]^{-1}=\left[1+2\left(\frac{f \rho}{t}\right)+\left(\frac{f \rho}{t}\right)^{2}\right]^{-1}=\left[1+\left(\frac{f \rho}{t}\right)\right]^{-2}
$$

If the patient is without any treatment then $t 1=t 2=1$ and under that condition, the probability of a person is healthy with equal correlated illnesses factors, $f$, is depicted in Fig. 7. 


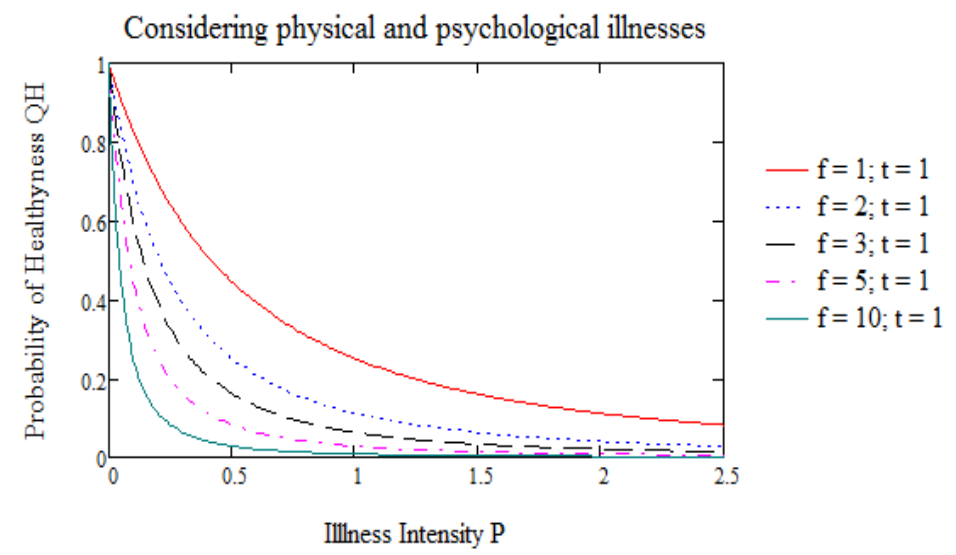

Fig. 7: The healthiness probability with different values of equal correlated factors, $f$.

Now consider another special condition, where the illnesses are happening in uncorrelated manner. Then we can write $\alpha=\beta=f=1$. Setting these values into Eq. (16), the probability that a person will be healthy with equal physical and psychological illnesses, uncorrelated manner illnesses, but with equal treatment factors $t 1=t 2=t$ is

$$
Q_{H}=\left[1+\left(\frac{\rho}{t}\right)\right]^{-2}
$$

The numerical results with normal treatment is $\mathrm{p}$ [resented in Figure 8 (a), with different values of equal treatment factors, and the illnesses are happening in uncorrelated manner. However, in the verge of Internet of bio-nano things, illnesses cannot happen in uncorrelated manner. Certainly, $\alpha=\beta=f$ will be greater than one.
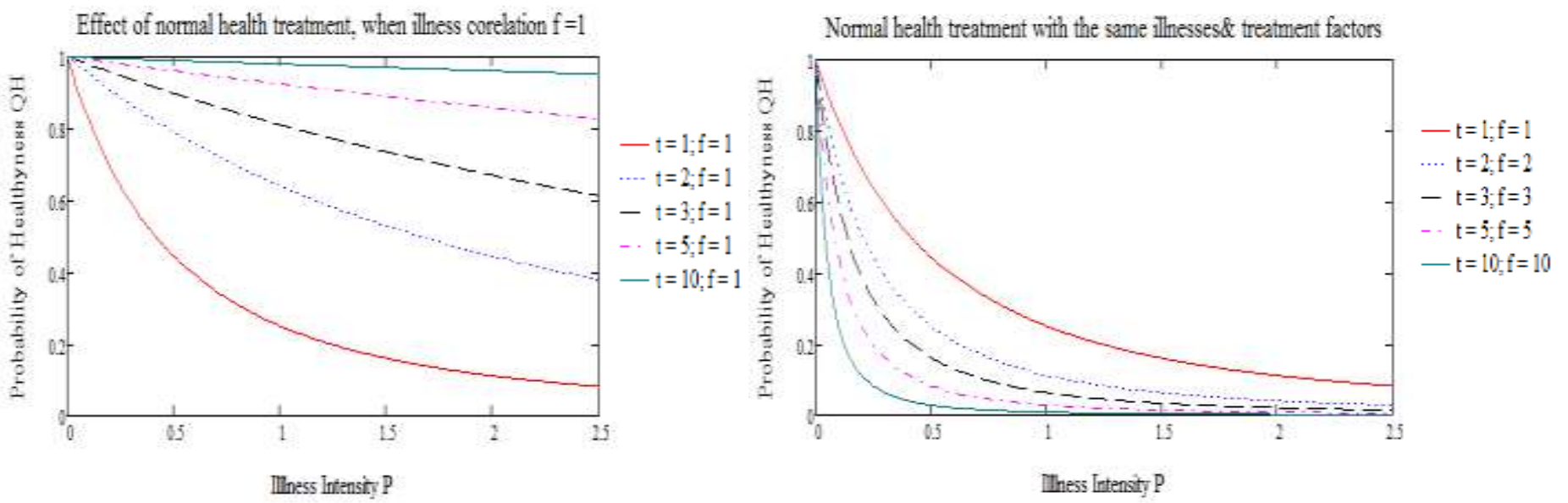

Fig. 8: (a) The healthiness probability with normal treatment having different values of treatment factors, $t$; (b) The healthiness probability with normal treatment having complex life style.

\subsection{The Probability of Healthiness with higher complexity of life}

Due to the increased complexity of life, it is expected that in next ten years, the illness correlations factors will be at least two folds. It means, with the equal illness correlations factors we may write $\alpha^{2}=\beta^{2}=f^{2}$. Let us consider another special case, where the illnesses correlation factors are equals, but more than one. For example, $f=1,2,3,5$ and 10 . With traditional normal treatment, having equal treatment correlation factors, but the values are more than one. For example, $t 1=t 2=t=1,2,3,5$ and 10 . If we use these in Eq. (16) then the healthiness probability is 


$$
Q_{H}=\left[1+\left(\frac{f^{2} \rho}{t}\right)\right]^{-2}
$$

The numerical results of Eq. (18) are depicted in Figure 8 (b). Obviously, the tradition treatment needs some changes, since it cannot provide the higher healthiness probability with high illness intensity. Most probably, nanotechnology based treatment Internet of bio-nano things based treatment is the solution.

\subsection{The Internet of Bio-nano Things based Treatment with Higher complex Life}

Consider that the probability of a person is healthy using nanotechnology based treatment is $Q_{H n}$. Let us consider first a person will become physically ill first with a probability of $Q_{P n}$ (Figure 9). Due to the higher complexity of life, a person will become physically ill with a higher rate. Therefore, the rate of a person will become physically ill with a rate of $\alpha^{2} \lambda 1$ instead of $\alpha \lambda 1$. The nanotechnology based treatment can achieve maximal treatment efficacy [5]. Considering the nanotechnology based treatment, the rate of a person will become physically ill with a rate of $\frac{\alpha^{2}}{n 1^{2}} \lambda 1$ instead of $\frac{\alpha}{n 1} \lambda 1$. The minimum side effect is another big advantage of the nanotechnology based treatment [5]. Therefore, we can assume a higher recovering rate with nanotechnology based treatment. Thus, the recovering rate is $n 1 \mu 1$, instead of $\mu 1$.

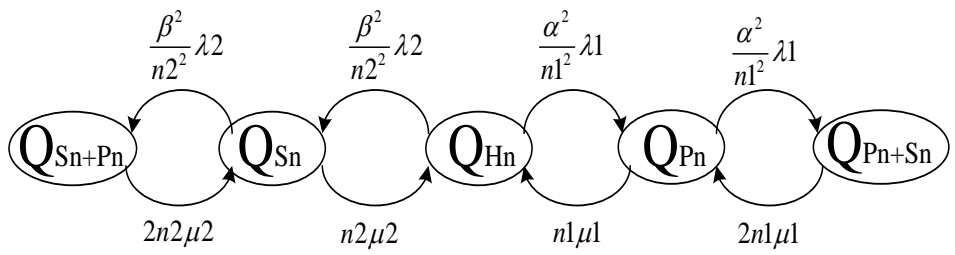

Fig. 9: The probability of healthiness, considering complex life style and Internet of bio-nano things based treatment.

The probability that a person will become physically ill first with higher complexity of life and nanotechnology based treatment can be written as

$$
Q_{P n}=Q_{H n} \frac{\alpha^{2} \lambda 1}{n 1^{2}} \frac{1}{n 1 \mu 1}=Q_{H n} \frac{\alpha^{2} \rho 1}{n 1^{3}}
$$

The probability that a person will become physically ill first then also psychologically ill with higher complexity of life and nanotechnology based treatment is

$$
Q_{P_{n}+S_{n}}=Q_{P_{n}} \frac{\alpha^{2} \lambda 1}{n 1^{2}} \frac{1}{2 n 1 \mu 1}=Q_{P_{n}} \frac{\alpha^{2} \rho 1}{2 n 1^{3}}=Q_{H n} \frac{\alpha^{2} \rho 1}{n 1^{3}} \frac{\alpha^{2} \rho 1}{2 n 1^{3}}=Q_{H n} \frac{1}{2}\left(\frac{\alpha^{2} \rho 1}{n 1^{3}}\right)^{2}
$$

The probability that a person will become psychologically ill first with higher complexity of life and nanotechnology based treatment can be written as

$$
Q_{S n}=Q_{H n} \frac{\beta^{2} \lambda 2}{n 2^{2}} \frac{1}{n 2 \mu 2}=Q_{H n} \frac{\beta^{2} \rho 2}{n 2^{3}}
$$

The probability that a person will become psychologically ill first then also physically ill with higher complexity of life and nanotechnology based treatment is

$$
Q_{S n+P n}=Q_{S n} \frac{\beta^{2} \lambda 2}{n 2^{2}} \frac{1}{2 n 2 \mu 2}=Q_{S n} \frac{\beta^{2} \rho 2}{2 n 2^{3}}=Q_{H n} \frac{\beta^{2} \rho 2}{n 2^{3}} \frac{\beta^{2} \rho 2}{2 n 2^{3}}=Q_{H n} \frac{1}{2}\left(\frac{\beta^{2} \rho 2}{n 2^{3}}\right)^{2}
$$


In steady state, the addition of all five probabilities shown in Fig. 7 is equals to one. Mathematically, we can write

$$
Q_{H n}+Q_{P n}+Q_{P n+S n}+Q_{S n}+Q_{S n+P n}=1
$$

Using the values given in Eqs. (9) to (12) and setting those values into Eq. (13), we can evaluate the probability that a person will healthy and can be written as

$$
Q_{H n}=\left[1+\left(\frac{\alpha^{2} \rho 1}{n 1^{3}}\right)+\frac{1}{2}\left(\frac{\alpha^{2} \rho 1}{n 1^{3}}\right)^{2}+\left(\frac{\beta^{2} \rho 2}{n 2^{3}}\right)+\frac{1}{2}\left(\frac{\beta^{2} \rho 2}{n 2^{3}}\right)^{2}\right]^{-1}
$$

To simplify the Eq. (24), let us consider that the physical and psychological illnesses correlation factors are equals. Thus, it can be written as $\alpha=\beta=f$. Assuming the nano-technological treatment correlation factors in equals then we can write $n t 1=n t 2=n$. Setting these two values in Eq. (24), the simplified probability that a person is healthy, with Internet of bio-nano based treatment and enjoying a complex life is

$$
Q_{H n}=\left[1+\left(\frac{f^{2} \rho}{n^{3}}\right)+\frac{1}{2}\left(\frac{f^{2} \rho}{n^{3}}\right)^{2}+\left(\frac{f^{2} \rho}{n^{3}}\right)+\frac{1}{2}\left(\frac{f^{2} \rho}{n^{3}}\right)^{2}\right]^{-1}=\left[1+2\left(\frac{f^{2} \rho}{n^{3}}\right)+\left(\frac{f^{2} \rho}{n^{3}}\right)^{2}\right]^{-1}=\left[1+\left(\frac{f^{2} \rho}{n^{3}}\right)\right]^{-2}
$$

The probability of healthiness using the Internet of bio-nano things is shown in Figure 10. Clearly, the future Internet of bio-nano things based treatment is essential, when the life will be more complex. Figure 8 (b) and Figure 10 show the clear difference between the normal treatment and the nano-technological based treatment, when the life will be complex.

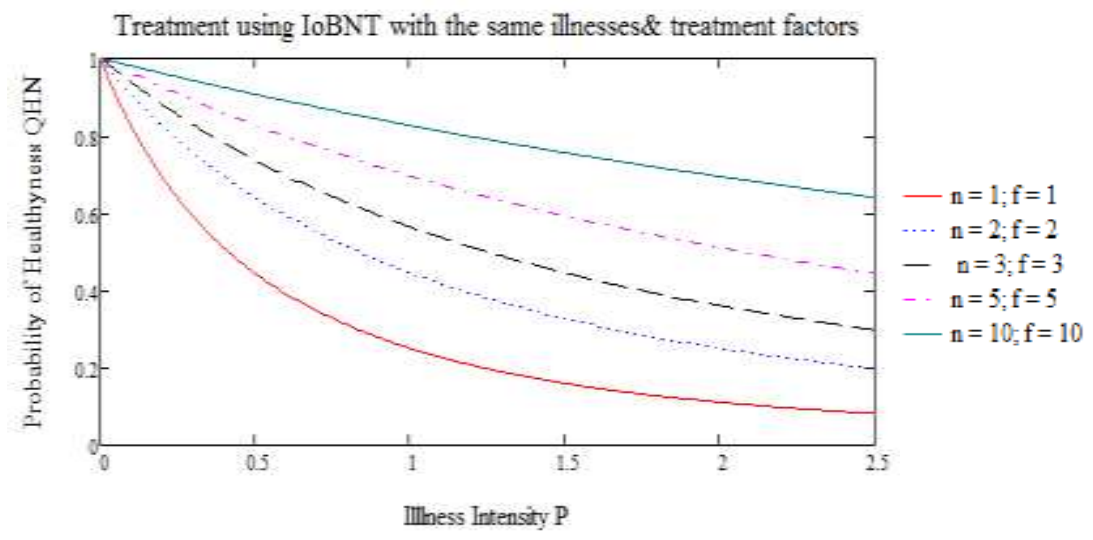

Fig. 10: The enhanced healthiness probability with IoBNT treatment having different values of treatment factors, $n$ and equal illness

\section{Conclusion} factors $f$.

Because of the increased complexity of lifestyle, the rate of a person becoming ill will increase. The traditional health treatment system will be unable to to keep up with the rapid transitions and changes of lifestyle choices that will result in impacts on the healthiness of an individual and thus improve the healthiness probability as shown in Figure 8 (b). On the other hand, a nanotechnology based treatment, such as Internet of bio-nano things based treatment can improve the healthiness probability tremendously despite the major instigations of physical and psychological illnesses and their intensities that will accompany the changes of people's' lifestyles (as depicted in Figure 10).

\section{References}

[1] I. F. Akyildiz, M. Pierobon, S. Balasubramaniam, and Y. Koucheryavy, "The Internet of Bio-Nano Things," IEEE Communications Magazine, vol. 53, no. 3, pp. 32 - 40, March 2015. 
[2] Y. Chahibi, M. Pierobon and I. F. Akyildiz, "Pharmacokinetic Modeling and Biodistribution Estimation Through the Molecular Communication Paradigm," IEEE Transactions on Biomedical Engineering, vol. 62, no. 10, pp. 2410 2420, October 2015.

[3] Youssef Chahibi, Ian F. Akyildiz and Sasitharan Balasubramaniam, "Molecular Communication Modeling of Antibody-Mediated Drug Delivery Systems," IEEE Transactions on Biomedical Engineering, vol. 62, no. 7, pp. 1683 -1695 , July 2015.

[4] A. C. Bonoiu, S. D. Mahajan, Hong Ding, Indrajit Roy, Ken-Tye Yong, Rajiv Kumar, Rui Hu, Earl J. Bergey, Stanley A. Schwartz, and Paras N. Prasad, "Nanotechnology approach for drug addiction therapy: Gene silencing using delivery of gold nanorod-siRNA nanoplex in dopaminergic neurons," Proc. Natl. Acad. Sci. U.S.A., vol. 106, no. 14, pp. 5546-5550, March 2009.

[5] S. K. Sahoo, S. Parveen and J. J. Panda, "The present and future of nanotechnology in human health care," Nanomedicine: Nanotechnology, Biology, and Medicine, vol. 3, pp. 20-31, 2007.

[6] Trisha Van Zandt and James T. Townsend, "Mathematical Psychology," in APA Handbook of Research Methods in Psychology: Research Designs: Quantitative, Qualitative, Neuropsychological, and Biological, P. M. Camic, D. L. Long, A. T. Panter, D. Rindskopf, H. Cooper, K. J. Sher, Eds. Vol. 2, Chapter 20, pp. 369-386. 